\title{
Learning Analytics em processos de personalização de aprendizagem: uma revisão sistemática de literatura
}

\author{
Aline de Campos ${ }^{1}$, Sílvio César Cazella ${ }^{12}$ \\ ${ }^{1}$ Programa de Pós-Graduação em Informática na Educação - Universidade Federal do \\ Rio Grande do Sul (UFRGS) \\ ${ }^{2}$ Programa de Pós-Graduação em Ensino na Saúde - Universidade Federal de Ciências \\ da Saúde de Porto Alegre (UFCSPA) \\ alinedecamposegmail.com, silvioceufcspa.edu.br
}

Resumo: A área de Learning Analytics (LA) tem sido proeminente na última década no sentido de ampliar as possibilidades de coleta, uso e análise de dados na educação. Este artigo apresenta a concepção, condução e resultados de uma revisão sistemática com objetivo de identificar métodos e recursos, benefícios e desafios de LA, sobretudo em processos de personalização da aprendizagem. Seguindo um protocolo de revisão, fezse a busca por artigos de língua inglesa publicados em periódicos entre 2013 e 2018 e chegou-se a seleção de 12 estudos que foram analisados em profundidade. Os principais resultados demonstram que existem claros relacionamentos de LA com processos de personalização, sobretudo no desenvolvimento de sistemas adaptativos. Entretanto, a área ainda guarda muitos desafios relativos a superação da complexidade técnica, reflexão das questões éticas, estudos do papel do julgamento humano e da validação de LA nos mais diversos cenários educacionais.

Palavras-chave: learning analytics, personalização, aprendizagem, revisão sistemática.

\section{Learning Analytics in processes of personalized learning: a systematic literature review}

\begin{abstract}
The Learning Analytics (LA) area has been prominent in the last decade in the sense of expanding the possibilities of collect, use and analysis of educational data. This article presents the design, conduct and results of a systematic review with the objective of identifying methods and resources, benefits and challenges of LA, especially in personalized learning process. Following a review protocol, we selected English language articles published in journals between 2013 and 2018 and 12 studies were analyzed in depth. The main results demonstrate that there are clear connection of LA with personalized learning, mostly in the development of adaptive systems. However, the area still have many challenges related to overcoming technical complexity, to ethic issues, the role of human judgment and the validation of LA in the most diverse educational scenarios.
\end{abstract}

Key-Words: learning analytics, personalization, learning, systematic review.

\section{INTRODUÇÃO}

O processo de Learning Analytics (LA) busca coletar, medir, analisar e relatar os dados e seus contextos com objetivo de otimizar o aprendizado e o ambiente em que este ocorre (Moissa et al., 2015). Apresenta ciclos que consideram a participação humana e procura-se entender o contexto como um todo, numa abordagem holística. $\mathrm{O}$ alinhamento de Learning Analytics voltado para melhorias na tomada de decisão se 
mostra favorável aos processos de personalização na educação. Percebe-se neste processo que os alunos obtêm "abordagens customizadas para auxiliá-los a aprender e base de informação em um método que se encaixe às suas necessidades e não a classe toda" (Chamberlin, 2016).

As tendências em educação caminham para uma aprendizagem diferenciada. Inicia-se uma mudança gradual, movendo a educação de uma "experiência homogênea de um para muitos para uma experiência de aprendizagem personalizada e profundamente imersiva" (King et al., 2016). Nisso as tecnologias de informação e comunicação são fundamentais e a informática na educação cresce em seu papel.

Há espaço para Learning Analytics no sentido de aprofundar possibilidades de uso de dados na educação numa perspectiva que contemple o novo perfil de alunos. Existe a crescente demanda da necessidade de uma variação dos métodos e ferramentas utilizados "para avaliar, medir e documentar a prontidão acadêmica, o progresso de aprendizagem, a aquisição de habilidades e outras necessidades educacionais dos estudantes" (Adams Becker et al., 2017).

Tendo em vista este cenário, através da realização de uma revisão sistemática de literatura, busca-se mapear estudos que correlacionam as práticas de Learning Analytics com processos de personalização na educação.

\section{MÉTODO DE REVISÃO SISTEMÁTICA}

Segundo Kitchenham (2004) uma revisão sistemática de literatura consiste na identificação, avaliação e interpretação de estudos relevantes a uma questão de pesquisa específica, área de estudo ou fenômeno de interesse. Sendo assim, trata-se de um estudo secundário que faz uso da sistematização de estudos primários. Ainda segundo a autora, existem diversas razões para realização de um estudo desta natureza, tais como: sumarizar evidências relativas a um assunto, identificar lacunas em uma pesquisa a fim de sugerir áreas para investigações futuras, bem como prover fundamentação no posicionamento para novas atividades de pesquisa.

A estruturação da revisão sistemática adotada neste estudo segue uma composição dos processos propostos por Kahn et al. (2000), Kitchenham (2004), Petersen et al. (2008) e Higgins e Green (2008) conforme apresentados na Figura 1.
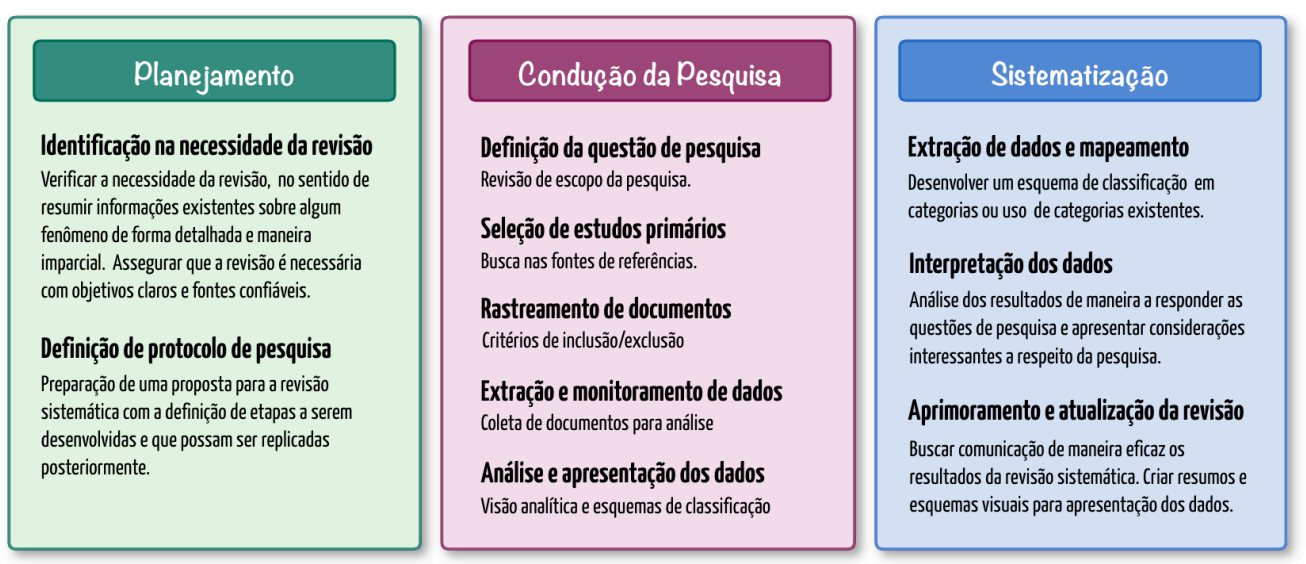

Figura 1 - Estrutura da revisão sistemática adota neste estudo.

\subsection{Planejamento}

A seguir busca-se analisar a necessidade de realização desta revisão sistemática 
tendo em vista trabalhos relacionados, bem como descreve-se o protocolo de pesquisa definidos as etapas e procedimentos realizados no processo.

\subsubsection{Necessidade da pesquisa}

Embora existam revisões sistemáticas com a temática de Learning Analytics, identificou-se que não há direcionamento a aplicação em processos de personalização da aprendizagem. Sendo esta uma tendência no cenário educacional, tanto em processos online, quanto presenciais, acredita-se relevante o entendimento de suas possíveis integrações com LA.

Assim, o objetivo deste estudo é mapear as correlações entre Learning Analytics e processos de personalização da aprendizagem, através de uma revisão sistemática da produção de artigos científicos publicados em periódicos em língua inglesa entre os anos de 2013 e 2018 tendo como foco aplicações em cenários educacionais.

\subsubsection{Protocolo de pesquisa}

Conforme demonstrado na Figura 1 anteriormente apresentada, o processo de revisão definido para este projeto inicia-se com a fase de planejamento onde se identifica a necessidade de realização da revisão, devendo-se reconsiderar sua execução caso haja outros estudos de mesmo direcionamento. Após, deve-se definir um protocolo de pesquisa com rigor metodológico, a fim de que o experimento possa ser replicado por outros pesquisadores.

Passa-se então para a fase de condução onde se definem as questões norteadoras que vão delimitar o escopo de análise. A seleção dos estudos primários conduz a busca nas bases de dados científicas para identificar as palavras-chave de relevância. Definem-se critérios de inclusão e de exclusão para o refinamento da busca, chegando a seleção de recursos a serem avaliados na etapa de análise e classificação.

Por fim, na fase de sistematização se realiza o mapeamento dos aspectos relevantes dos estudos selecionados tendo em vista as questões norteadoras. Neste momento pode-se criar esquemas de classificação para os dados, no sentido de agrupar elementos correlatos. Posteriormente, a partir de uma visão analítica busca-se responder as questões, bem como realizar reflexões e relatar os resultados da pesquisa.

\subsection{Condução da pesquisa}

A seguir apresentam-se os processos de definição de questões norteadoras da pesquisa, a seleção dos estudos primários e os procedimentos metodológicos referentes ao rastreamento, seleção, monitoramento e apresentação dos dados.

\subsubsection{Definição de questões de pesquisa}

Foram definidas cinco questões que nortearam o escopo da pesquisa. Em âmbito geral (Q1) buscou analisar de que forma Learning Analytics se relaciona com os processos de personalização de aprendizagem. Como questões secundárias, buscou-se verificar quais são os métodos e recursos mais utilizados neste âmbito, bem como os benefícios e desafios que o uso de Learning Analytics pode apresentar (Q2, Q3 e Q4). Por fim, apresenta-se uma questão que busca verificar assuntos relacionados a temática no sentido de expandir as possibilidades de pesquisas futuras (Q5). O Quadro 1 apresenta a formulação das questões. 
Quadro 1 - Questões norteadoras da revisão sistemática.

\begin{tabular}{|l|l|}
\hline ID & QUESTÃo \\
\hline Q1 & De que forma Learning Analytics se relaciona com processos de personalização da aprendizagem? \\
\hline Q2 & Quais são os métodos e recursos usados em Learning Analytics? \\
\hline Q3 & Quais são os benefícios do uso de Learning Analytics? \\
\hline Q4 & Quais são os desafios no uso de Learning Analytics? \\
\hline Q5 & Quais assuntos estão relacionados com Learning Analytics e personalização de aprendizagem? \\
\hline
\end{tabular}

\subsubsection{Seleção de estudos primários}

Foram escolhidas como fontes de consulta as bases Scopus (Elsevier) ${ }^{1}$, Web of Science (Thomson Reuters/Clarivate) ${ }^{2}$ e Springer Link (Springer International Publishing $)^{3}$, tendo em vista sua relevância e vasta quantidade de títulos disponíveis. As palavras chaves foram escolhidas após alguns testes de retorno nas bases. Inicialmente trabalhou-se com os termos "learning analytics" e "personalization", porém ao analisar alguns retornos percebeu-se a ampla utilização do termo "personalized learning", sendo assim agregou-se na string de busca, resultando em: ("learning analytics" AND ("personalized learning" OR "personalization")).

\subsubsection{Rastreamento de documentos}

Os critérios de inclusão e exclusão foram elaborados no sentido de criar um escopo viável de pesquisa e selecionar um conjunto de recursos capazes de auxiliar nas respostas das questões elaboradas. O Quadro 2 apresenta os critérios definidos.

Quadro 2 - Critérios de inclusão e de exclusão

\begin{tabular}{|c|c|}
\hline CRITÉRIOS DE INCLUSÃO & CRITÉRIOS DE EXCLUSÃO \\
\hline $\begin{array}{l}\text { [I1] Artigo publicados em periódicos. } \\
\text { [12] Artigo publicado entre os anos de } 2013 \text { e } 2018 . \\
\text { [13] Artigo em língua inglesa. } \\
\text { [14] Artigo que menciona processos de personalização } \\
\text { de aprendizagem. }\end{array}$ & $\begin{array}{l}\text { [E1] Artigo duplicado. } \\
\text { [E2] Não apresenta acesso gratuito ao texto completo. } \\
\text { [E3] Não é um artigo embora esteja em um periódico. } \\
\text { [E4] Poucas relações com processos de personalização de } \\
\text { aprendizagem. }\end{array}$ \\
\hline
\end{tabular}

Tendo em vista o desejo de selecionar estudos de boa qualidade, focou-se em artigos publicados em periódicos com revisão por pares [I1]. Mapeou-se trabalhos mais recentes na área entre os anos de 2013 e 2018 [I2]. Por uma questão de abrangência selecionou-se artigos em língua inglesa [I3]. Por fim, obviamente, delimitou-se que os artigos deveriam mencionar relações com personalizaçao de aprendizagem [I4].

Em relação os critérios de exclusão, [E1] diz respeito a artigos que foram apresentados nas buscas em mais de uma base de dados. Já o critério de exclusão [E2], indica a impossibilidade de acesso gratuito ao texto original completo. Percebeu-se que na seleção alguns textos tratavam-se de revisões de livros, bem como de textos escritos por editores de periódicos, estes então foram rejeitados seguindo o critério de exclusão [E3]. Para análise do critério de exclusão [E4], além da análise dos resumos (abstract), fez-se a leitura diagonal considerando a introdução, principais tópicos e considerações finais, buscando identificar se estes apresentavam relacionamento entre Learning Analytics e personalização da aprendizagem. Nesse processo, houve exclusão de artigos

\footnotetext{
Acesso em: https://www.scopus.com

${ }^{2}$ Acesso em: https://webofknowledge.com

${ }^{3}$ Acesso em: https://link.springer.com
} 
por tratarem-se de revisão integrativa ou sistemática, por estarem fora do contexto dessa pesquisa e outros por abordarem apenas brevemente a questão de personalização ou estarem focados na apresentação técnica de um solução de LA e não na aplicação em cenários educacionais.

O Quadro 3 apresenta a quantidade de artigos verificados na busca geral, ou seja, sem critérios de seleção por tipo de conteúdo, fonte de dados ou período. Após, apresenta-se a quantidade de artigos filtrando pelos critérios de inclusão estabelecidos e posteriormente apresenta-se a aplicação de cada um dos critérios de exclusão e a quantidade de artigos resultados. Ressalta-se que no caso da base Springer Link, que possui categorizações pré-definidas, fez-se o filtro também pela categoria "Learning \& Instruction" a fim de atender aos critérios estabelecidos.

Quadro 3 - Quantidade de artigos e aplicação de filtros

\begin{tabular}{|c|c|c|c|c|c|c|}
\hline & \multirow{2}{*}{$\begin{array}{l}\text { BUSCA GERAL } \\
\text { Sem filtros por } \\
\text { período, tipo ou } \\
\text { fonte de dados }\end{array}$} & \multirow{2}{*}{$\begin{array}{l}\text { FILTROS APLICADOS } \\
\text { Artigos publicados } \\
\text { em periódicos entre } \\
2013 \text { e } 2018\end{array}$} & \multicolumn{4}{|c|}{ CRITÉRIOS DE EXCLUSÃO } \\
\hline & & & $\begin{array}{c}\text { [E1] } \\
\text { Artigos } \\
\text { duplicados }\end{array}$ & $\begin{array}{c}\text { [E2] } \\
\text { Impossibilidade } \\
\text { de acesso }\end{array}$ & $\begin{array}{c}\text { [E3] } \\
\text { Não é um artigo } \\
\text { científico }\end{array}$ & $\begin{array}{c}\text { [E4] } \\
\text { Sem foco em } \\
\text { personalização }\end{array}$ \\
\hline Scopus & 100 & 19 & 19 & 9 & 9 & 7 \\
\hline Web of Science & 53 & 14 & 3 & 2 & 2 & 1 \\
\hline Springer Link & 477 & 21 & 21 & 21 & 16 & 4 \\
\hline TOTAL & 630 & 54 & 43 & 32 & 27 & 12 \\
\hline
\end{tabular}

\subsubsection{Extração e monitoramento de dados}

Aplicados os critérios de inclusão e exclusão chegou-se a 12 artigos selecionados e passou-se a leitura completa e aprofundada dos materiais. Neste processo, avaliou-se a qualidade dos estudos selecionados, no sentido de analisar se estes apresentavam: a) estrutura adequada; b) definição de métodos utilizados; c) fundamentação teórica e referências relevantes; d) reflexões acerca da temática.

O Quadro 4 apresenta na primeira coluna o identificador que será adotado para menção dos estudos nesta revisão, a segunda coluna refere-se ao tipo de estudo: [EXP] artigos que apresentam relato de experimentos nos mais diversos âmbitos educacionais; [REF] reflexão analítica, estudos com bom referencial e reflexões acerca das temáticas. $\mathrm{Na}$ terceira coluna apresenta-se a referência completa dos artigos selecionados.

Quadro 4 - Conjunto de artigos selecionado para revisão

\begin{tabular}{|c|c|l|}
\hline ID & TIPO & REFERÊNCIA \\
\hline A01 & EXP & $\begin{array}{l}\text { BEEMER, J.; SPOON, K.; FAN, J.; STRONACH, J.; FRAZEE, J.P.; BOHONAK, A.J.; LEVINE, R. A. Assessing Instructional Modalities: } \\
\text { Individualized Treatment Effects for Personalized Learning, Journal of Statistics Education, 26:1, p. 31-39, 2018. }\end{array}$ \\
\hline A02 & REF & $\begin{array}{l}\text { AGUILAR, S.J. Learning Analytics: at the Nexus of Big Data, Digital Innovation, and Social Justice in Education. TechTrends, 62 } \\
(1), \text { p. 37-45, 2018. }\end{array}$ \\
\hline A03 & EXP & $\begin{array}{l}\text { ROBERTS, L.; HOWELL, J.; SEAMAN, K. Give Me a Customizable Dashboard: Personalized Learning Analytics Dashboards in } \\
\text { Higher Education. Technology, Knowledge and Learning, 22 (3), p. 317-333, 2017. }\end{array}$ \\
\hline A04 & EXP & $\begin{array}{l}\text { LIU, M.; KANG, J.; ZOU, W.; LEE, H.; PAN, Z.; CORLISS, S. Using Data to Understand How to Better Design Adaptive Learning. } \\
\text { Technology, Knowledge and Learning, 22 (3), p. 271-298, 2017. }\end{array}$ \\
\hline A06 & EXP & $\begin{array}{l}\text { BALLARD, J.; BUTLER, P. I. Learner enhanced technology: can activity analytics support understanding engagement a } \\
\text { measurable process? Journal of Applied Research in Higher Education, Vol. 8, Issue: 1, p.18-43, 2016. }\end{array}$ \\
\hline A07 & REF & $\begin{array}{l}\text { DE FREITAS, S.; GIBSON, D.; DU PLESSIS, C.; HALLORAN, P.; WILLIAMS, E.; AMBROSE, M.; DUNWELL, I.; ARNAB, S. Foundations } \\
\text { 46 (6), p. 1175-1188, 2015. }\end{array}$ \\
\hline A08 & EXP & $\begin{array}{l}\text { BAALSRUD-HAUG, } \\
\text { KIILI, K.; NINAUS, M.; FREITAS, S.; MAZZETTI, A.; DAHLBOM, A.; DEGANO, C. Learning Analytics Architecture to Scaffold }\end{array}$ \\
\hline
\end{tabular}




\begin{tabular}{|c|c|l|}
\hline & & Learning Experience through Technology-based Methods. International Journal of Serious Games. v.2 Issue 1. p. 29-44, 2015. \\
\hline A09 & REF & REYES, J.A. The skinny on big data in education: learning analytics simplified. TechTrends. 59, 2015. \\
\hline A10 & EXP & $\begin{array}{l}\text { MARTIN, F.; WHITMER, J. C.. Applying Learning Analytics to Investigate Timed Release in Online Learning. Technology, } \\
\text { Knowledge and Learning, v.21, 1, p. 59, 2016. }\end{array}$ \\
\hline A11 & EXP & $\begin{array}{l}\text { IFENTHALER, D.; WIDANAPATHIRANA, C. Development and Validation of a Learning Analytics Framework: Two Case Studies } \\
\text { Using Support Vector Machines. Technology, Knowledge and Learning, v.19, 1-2, p. 221, 2014. }\end{array}$ \\
\hline A12 & EXP & GIBSON, D.; FREITAS, S. Exploratory Analysis in Learning Analytics. Technology, Knowledge and Learning, v. 21, 1, p. 5, 2016. \\
\hline
\end{tabular}

\subsubsection{Análise e apresentação dos dados}

As questões norteadoras foram consideradas na análise dos artigos, onde criouse a sistematização dos principais conteúdos associados nas questões Q1, Q2, Q3 e Q4. Para resposta à questão norteadora Q5, na leitura de cada artigo foram estabelecidas palavras-chave adicionais, para além das que o artigo apresentava. Fez-se uso também de processo de mineração de textos no sentido de verificar a análise de frequência de termos, bem como associações possíveis.

\section{ANÁLISE E DISCUSSÃO DOS RESULTADOS}

Dentro do protocolo estabelecido para a revisão, apresenta-se a etapa de sistematização. Neste sentido, realizou-se a extração de dados e mapeamento criando esquemas de classificação e categorias buscando realizar a interpretação de dados e procurando responder às questões norteadoras previamente definidas. A seguir apresentam-se as principais análises e reflexões acerca do assunto.

\subsubsection{Correlações entre Learning Analytics e personalização da aprendizagem}

Todos os trabalhos selecionados para análise apresentam indícios de como LA relaciona-se com processos de personalização de aprendizagem. O uso de LA para proporcionar experiências personalizadas e estratégias pedagógicas mais assertivas para os alunos é bastante mencionado [A02; A04; A01]. Siemens e Baker (2012), precursores nos estudos de LA, reforçam esta questão e ressaltam sua natureza multifacetada que vai além da recomendação de conteúdos, tratando sobretudo de incorporar tecnologia em conjunto com socialização e pedagogia [A07; A12]. Além disso, surge a questão das oportunidades educacionais com base nas necessidades e capacidades de cada aluno a partir da elaboração de perfis e indicações de intervenções personalizadas que levem em consideração o contexto.

Verifica-se o uso do termo "aprendizagem adaptativa" como sinônimo de personalização de aprendizagem [A04; A10; A08] onde atende-se a necessidade do desenvolvimento de sistemas de instruções personalizadas que podem ser capazes de permitir abordagens adaptativas [A02; A12]. Isto certamente amplia as possibilidades de melhoria na experiência de aprendizagem dos alunos, uma vez que os docentes podem ter acesso a sua jornada prévia [A06; A10]. Sendo assim parte-se para um processo de atenção individualizada e não a tradicional condução dos processo voltados para a média [A02]. Ainda, aponta-se para os processo de LA como importante apoio ao docente, por tratar-se do condutor da experiência do aluno [A08].

Alguns trabalhos mencionam o aumento do engajamento dos alunos nos mais diversos níveis: comportamental, emocional e cognitivo [A05], bem como na motivação [A02] e retenção dos alunos [A06] provendo insights aos docentes e o desenvolvimento de experiências de aprendizagem mais interativas. Recursos muito apontados pelos trabalhos analisados dão conta de processos de sugestão de materiais a partir de 
sistemas de recomendação [A04], agentes inteligentes [A02] e dashboards flexíveis [A03]. Outro ponto de atenção é a necessidade de que esses processos levem em consideração fortemente as questões de tempo e histórico. Ou seja, considerar o desempenho dos alunos ao longo do tempo [A06], avaliar seus aprendizados anteriores [A08], realizar adaptações em tempo real [A09; A11].

\subsubsection{Métodos e recursos usados em Learning Analytics}

Em relação aos métodos e recursos frequentemente usados para construção de processos de LA, fez-se um mapeamento das técnicas, aplicações e fontes de dados mencionadas nos artigos para revisão. As referências indicam multiplicidade de métodos e recursos combinados e mencionam data mining e big data como foco. $\mathrm{O}$ Quadro 5 apresenta a sistematização dos dados encontrados.

Quadro 5 - Métodos e recursos utilizados na construção de processos de LA

\begin{tabular}{|c|c|c|}
\hline CATEGORIA & ASSUNTO & ESTUDOS \\
\hline $\begin{array}{l}\text { Machine } \\
\text { learning }\end{array}$ & $\begin{array}{l}\text { Regressão linear e logística; Métodos lineares, não lineares e simbólicos; Modelos } \\
\text { lineares hierárquicos; Redes Bayesianas e neurais; Random Forest; Árvores de } \\
\text { decisão; Support vector machines (SVM); Lógica Fuzzy; Clusterização; }\end{array}$ & A01; A11; A04; A12; A05 \\
\hline Estatísticas & Métodos e cálculos estatísticos; & A03; A01 \\
\hline Visualização & Visualização de dados; Dashboards customizáveis; Sistemas de alerta; & A02; A03; A05; A09; A11 \\
\hline Textos & Mineração de textos; Processamento de Linguagem Natural; & A03; A07; A11; A02 \\
\hline Recomendação & Algoritmos de recomendação; & A04; A08; A09 \\
\hline Adaptação & $\begin{array}{l}\text { Navegação adaptativa; Sequenciamento de conteúdos; Personalização de } \\
\text { conteúdos e apresentação; Sistemas adaptativos e personalizáveis; }\end{array}$ & A04; A10; A08; A07 \\
\hline Social & $\begin{array}{l}\text { Análise de redes sociais; Mineração de relacionamentos; Modelagem de } \\
\text { conhecimento, comportamento e experiência do usuário; }\end{array}$ & A05; A07; A08 \\
\hline Predição & Modelos, monitoramento e predição; & A02; A07; A09; A10 \\
\hline $\begin{array}{l}\text { Origem dos } \\
\text { dados }\end{array}$ & $\begin{array}{l}\text { Sistemas de gerenciamento de aprendizagem; Sistemas gerenciais de educação; } \\
\text { Avaliação de alunos; Pesquisas e questionários; Metadados, Datasets educacionais; } \\
\text { Sistemas tutores inteligentes; Perfil de currículo; Tempo de acesso, frequência de } \\
\text { autenticação, costume de navegação, comportamento, interação com materiais; }\end{array}$ & A06; A05; A09; A11; A10 \\
\hline Métodos & $\begin{array}{l}\text { Agentes conversacionais; Animações; Análise de tendências; Sistemas tutores } \\
\text { inteligentes; Perfis de usuários; Comparativos; Modelagens; Descoberta com } \\
\text { modelos; Modelagem de domínios de conhecimento; }\end{array}$ & A11; A07; A08; A03; A06 \\
\hline
\end{tabular}

\subsubsection{Principais benefícios com o uso de Learning Analytics}

Acredita-se haver possibilidades de benefícios concretos advindos de processos de Learning Analytics para alunos, professores e instituições. A grande quantidade de dados dos estudantes, como informações demográficas, notas e comportamentos de alunos, amplia as possibilidades de estratégias de retenção e sucesso acadêmico, afastando-se assim do nivelamento pela média, para atender às necessidades de cada aluno de uma forma personalizada e orientada por dados [A02]. Pode-se detectar alunos que necessitam de auxílio no sentido de fornecer recursos e indicações de hábitos de estudo através de feedback personalizado e de forma prescritiva [A03].

Para os estudantes, os benefícios residem nas possibilidades de recebimento de atenção personalizada, ou seja, um design de aprendizagem focado em seu contexto e suas necessidades. O fornecimento de feedbacks auxilia no aumento da reflexão e consciência de sua trajetória de aprendizagem no sentido de engajar o aluno no processo de construção do seu conhecimento de forma crítica e ativa [A05; A03; A06]. 
Já para os docentes, percebe-se benefícios no sentido de apresentar informações a partir da detecção de padrões de comportamento, interação nos ambientes virtuais e aprendizagem dos estudantes que podem levar a melhores tomadas de decisão e ações estratégicas. Sendo assim, pode-se realizar intervenções que maximizam o potencial dos alunos na obtenção dos resultados de aprendizagem previstos [A09; A04; A01].

Do ponto de vista das instituições encaminha-se para a possibilidade de melhoria da qualidade dos cursos, tendo em vista processos de sequenciamento de currículos e alinhamento no desenvolvimento de competências, aprimoramento de materiais, melhorias pedagógicas e identificação de docentes de alto desempenho em comparativo a outros que precisam de assistência, bem como definir novas políticas institucionais e programas de desenvolvimento profissional [A11; A10; A09].

\subsubsection{Principais desafios com o uso de Learning Analytics}

Os grandes desafios do uso de LA associados aos processos de personalização são apresentados no conjunto de artigos analisados. Aponta-se que a maioria dos ambientes virtuais de aprendizagem usam uma abordagem de "tamanho único", sem um suporte personalizado [A10]. Sendo assim, há necessidade de chegar aos alunos de forma customizada [A02], tendo em vista contextos dinâmicos e em uma perspectiva sociocultural que leva em conta os conhecimentos anteriores [A05; A06].

Parecem faltar estudos relativos a aplicação de LA e sua aceitação nos mais diversos cenários, tanto por parte dos docentes, quanto dos alunos, numa perspectiva de validação dos perfis e as diferentes abordagens de análise [A03; A11]. Além disso, os docentes devem estar preparados para as novas abordagens com uso de LA, pois algumas modificações em suas práticas podem ser necessárias. Métodos já amplamente utilizados precisarão ser ajustados de acordo com as indicações da análise dos dados que vão demonstrar cenários diferentes de suas interpretações tradicionais [A09].

Do ponto de vista dos alunos, deve-se ter especial cuidado com as questões que dizem respeito aos comparativos que podem vir a ser realizados. Destaca-se que "professores tendem a favorecer aqueles que se comportam como o esperado e oferecem mais apoio àqueles com engajamento positivo" [A05], entretanto os alunos que demonstram necessidades diversas devem ter a devida atenção. Portanto, é importante oferecer formas de comparativos entre alunos, sem desmotivar ou desencorajar [A03].

Questões desafiadoras relativas aos aspectos técnicos na construção e gestão de sistemas que fazem uso de LA, tais como a grande quantidade de dados a serem processados [04], a extração de informações significativas dos dados complexos e heterogêneos, incluindo a necessidade de um produto final de fácil acesso e interpretação por parte dos usuários finais.

A partir do entendimento de que há grande complexidade no desenvolvimento e no processo de manter sistemas desta natureza, necessita-se de soluções escalonáveis e sustentáveis [A08]. Os maiores responsáveis por esta complexidade são a exigência de um suporte organizacional, bem como análise da qualidade dos dados, seu escopo e interoperabilidade [A07], sua grande heterogeneidade de formatos, necessidade de processamento em tempo real [A11], os processos de medição, coleta, análise, relatórios e compartilhamento de dados entre instituições [A09] e um design atraente e boa usabilidade na apresentação dos dados [A04].

Outro aspecto que merece especial atenção é o fato do amplo uso de dados pessoais que pode implicar em questões de privacidade e ética. Percebe-se essa preocupação em boa parte dos estudos analisados [A02; A03; A06; A07; A09].

Dentro destes processos a participação do capital humano se faz fundamental. 
Os projetos que envolvem LA só serão eficazes se tiverem um contexto holístico, com a capacidade de incluir dados adicionais por meio da observação e da manipulação dos conjuntos de dados [A07]. Assim, se faz fundamental incluir o aspecto humano e capacitá-lo para avaliar, interpretar e tomar decisões de qualidade baseadas em informações sistematizadas [A09, A10; A06] .

\subsubsection{Assuntos relacionados a temática de Learning Analytics e personalização}

A Figura 2 apresenta a sistematização dos principais assuntos dos artigos selecionados. A nuvem de termos demonstra principais tópicos extraídos a partir do processo de mineração de textos. Fez-se uso da ferramenta WordCounter ${ }^{4}$ para analisar a frequência dos termos tanto em palavra única, quanto em termos com duas e com três palavras. Assim, ao detectar os elementos mais frequentes, fez-se uma seleção que foi inserida no sistema Word $\mathrm{Art}^{5}$ onde criou-se a nuvem de termos ${ }^{6}$.

Figura 2 - Sistematização de conteúdos dos artigos selecionados

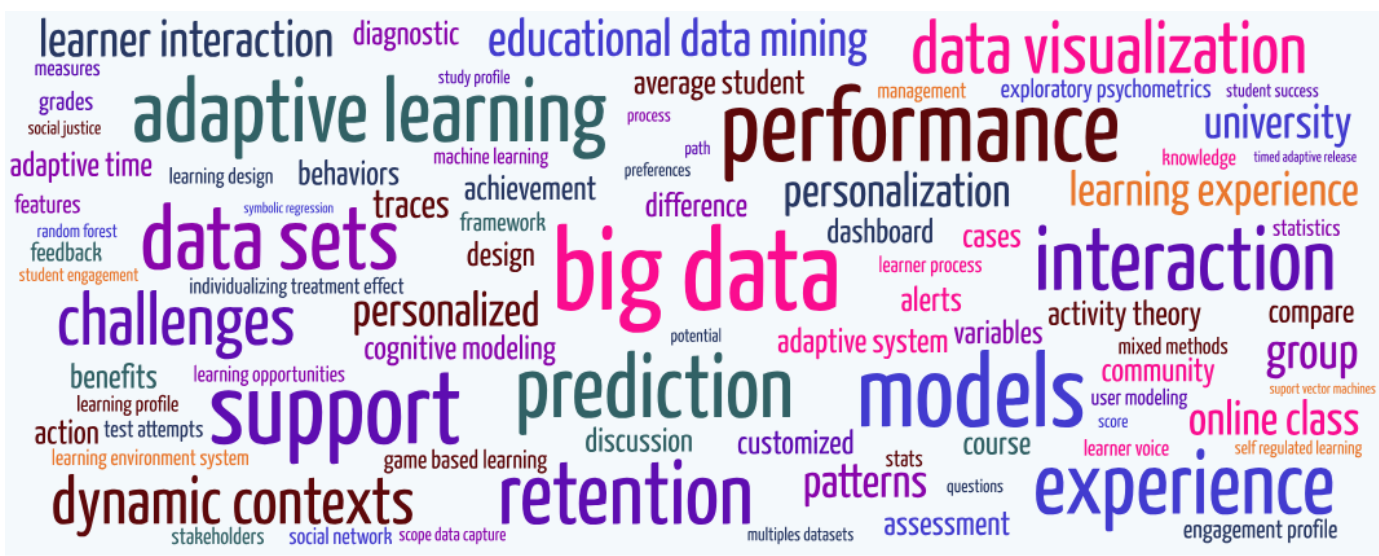

\section{CONSIDERAÇÕES FINAIS}

A revisão sistemática aqui apresentada, ocupou-se da análise das relações entre Learning Analytics e os processos de personalização de aprendizagem. Ao avaliar os artigos selecionados, percebeu-se um conjunto de ótimas reflexões acerca da temática em sua maioria apresentando experiências de aplicação em cenários reais. Entende-se que as reflexões não se esgotam no material selecionado, uma vez que dizem respeito a contextos específicos e que além disso, existem desafios nos processos técnicos de coleta, processamento e análise, bem como os processos operacionais que demandam entendimento dos envolvidos e adoção sistemática.

O potencial em processos de personalização fica claro uma vez que há crescente necessidade de atenção às necessidades individuais num processo inclusivo que leva em consideração o histórico e contexto dos alunos. Entretanto, percebe-se que estes processos são trabalhosos e não podem ser absorvidos apenas pela figura do professor, sob pena de aumento da já existente sobrecarga de trabalho. Assim, instituições devem auxiliar neste processo de desenvolvimento e implantação de recursos para este suporte, bem como na capacitação dos docentes e entendimento dos alunos do uso destes recursos com transparência e ética.

\footnotetext{
${ }^{4}$ Acesso em: https://wordcounter.net/

${ }^{5}$ Acesso em: https://wordart.com/create

${ }^{6}$ Como esperado o termo mais recorrente foi "Learning Analytics", por isso este foi retirado da seleção.
} 


\subsection{Trabalhos futuros}

Alguns pontos relevantes identificados como possibilidades de estudos futuros residem na integração de Learning Analytics com tecnologias cognitivas. Questões relativas à modelagem cognitiva como suporte importante aos processos voltados ao entendimento dos padrões de comportamento [A10], bem como as possibilidade de realização de modelagens psicométricas exploratórias e iterativas [A12].

Além disso, são apontadas possibilidades de integração de outros métodos já adotados em ambientes virtuais, tais como a gamificação e metodologias ativas, atenção às questões de user experience, dados multidimensionais e convergência de mídias, bem como a integração entre presencial e digital [A12]. Ainda o aprofundamento nos estudos de identificação dos parâmetros corretos para monitorar e avaliar a qualidade do processo de aprendizagem caminhando para a superação de complexidades técnicas.

Ainda parece existir uma grande lacuna entre a teoria e a prática no que diz respeito a Learning Analytics e as inúmeras possibilidades de modelos e estruturas, bem como a pouca integração das soluções já desenvolvidas. Sendo assim, existe um campo de estudos a serem explorados no sentido de integrar tecnologias, dados e tomada de decisão ampliando as possibilidades de processos de personalização de aprendizagem nos mais diversos âmbitos educacionais.

\section{REFERÊNCIAS}

ADAMS BECKER, S.; CUMMINS, M.; DAVIS, A.; FREEMAN, A.; HALL GIESINGER, C.; ANANTHANARAYANAN, V. NMC Horizon Report: 2017 Higher Education Edition. Austin, Texas: The New Media Consortium, 2017.

CHAMBERLIN, B. Cognitive Computing \& Education: HorizonWatch 2016 Emerging Trend Brief. IBM Market Development Insights, 2016.

HIGGINS, J.; GREEN, S. Cochrane Handbook for Systematic Reviews of Interventions Cochrane Book Series. A John Wiley \& Sons, Ltd Publication, 2008.

KING, M.; CAVE, R.; FODEN, M.; STENT, M. Personalised Education: from curriculum to career with cognitive systems. IBM Education. UK, 2016.

KITCHENHAM, B. Procedures for performing systematic reviews. Keele University, 33 (TR/SE-0401), 28, 2004.

KHAN, K.; TER, G.; GLANVILLE, J.; SOWDEN, J; KLEIJNEN, J. Undertaking Systematic Reviews of Research on Effectiveness. CRD's Guidance for Carrying Out or Commissioning Reviews. 2nd Edition. CRD Report No. 4. York: NHS Centre for Reviews and Dissemination, University of York, 2000.

MOISSA, B.; GASPARINI, I.; KEMCZINSKI, A. Educational Data Mining versus Learning Analytics: estamos reinventando a roda? Um mapeamento sistemático. In: Anais do XXVI Simpósio Brasileiro de Informática na Educação, 2015.

PETERSEN, K.; FELDT, R.; MUJTABA, S.; MATTSSON, M. Systematic Mapping Studies in Software Engineering. 12Th International Conference on Evaluation and Assessment in Software Engineering, 2008.

SIEMENS, G.; BAKER, R. Learning Analytics and Educational Data Mining: Towards Communication and Collaboration. In: Proceedings of the 2nd international conference on learning analytics and knowledge. ACM. p. 252-254, 2012. 